\title{
Recruitment and retention in obesity prevention and treatment trials targeting minority or low-income children: a review of the clinical trials registration database
}

\author{
Zhaohui Cui ${ }^{1}$, Elisabeth M. Seburg ${ }^{2}$, Nancy E. Sherwood ${ }^{2}$, Myles S. Faith ${ }^{1}$ and Dianne S. Ward ${ }^{1 *}$
}

\begin{abstract}
Background: Efforts to recruit and retain participants in clinical trials are challenging, especially in studies that include minority or low-income children. To date, no studies have systematically examined recruitment and retention strategies and their effectiveness in working successfully with this population. We examined strategies employed to recruit or retain minority or low-income children in trials that included an obesity-related behavior modification component.
\end{abstract}

Methods: First, completed home-, community-, and school-based trials involving minority or low-income children aged 2-17 years were identified in a search of the ClinicalTrials.gov registry. Second, a PubMed search of identified trials was conducted to locate publications pertinent to identified trials. Recruitment and retention rates were calculated for studies that included relevant information.

Results: Our final analytic sample included 43 studies. Of these, 25 studies reported recruitment or retention strategies, with the amount of information varying from a single comment to several pages; 4 published no specific information on recruitment or retention; and 14 had no publications listed in PubMed. The vast majority (92\%) of the 25 studies reported retention rates of, on average, $86 \%$. Retention rates were lower in studies that: targeted solely Hispanics or African Americans (vs. mixed races of African Americans, whites, and others); involved children and parents (vs. children only); focused on overweight or obese children (vs. general children), lasted $\geq 1$ year (vs. $<1$ year), were home or community-based (vs. school-based), included nutrition and physical activity intervention (vs. either intervention alone), had body mass index or other anthropometrics as primary outcome measures (vs. obesityrelated behavior, insulin sensitivity, etc.). Retention rates did not vary based on child age, number of intervention sessions, or sample size.

Conclusions: Variable amounts of information were provided on recruitment and retention strategies in obesityrelated trials involving minority or low-income children. Although reported retention rates were fairly high, a lack of reporting limited the available information. More and consistent reporting and systematic cataloging of recruitment and retention methods are needed. In addition, qualitative and quantitative studies to inform evidence-based decisions in the selection of effective recruitment and retention strategies for trials including minority or low-income children are warranted.

Keywords: African American, behavior, children, Hispanic, intervention, lifestyle, low income, recruitment, retention, systematic review

\footnotetext{
*Correspondence: dsward@email.unc.edu

'Department of Nutrition, Gillings School of Global Public Health, University of North Carolina at Chapel Hill, 2202 McGavran-Greenberg Hall, Campus Box 7461, Chapel Hill, NC 27599, USA

Full list of author information is available at the end of the article
}

\section{Ciomed Central}

(c) 2015 Cui et al. Open Access This article is distributed under the terms of the Creative Commons Attribution 4.0 International License (http://creativecommons.org/licenses/by/4.0/), which permits unrestricted use, distribution, and reproduction in any medium, provided you give appropriate credit to the original author(s) and the source, provide a link to the Creative Commons license, and indicate if changes were made. The Creative Commons Public Domain Dedication waiver (http://creativecommons.org/publicdomain/zero/1.0/) applies to the data made available in this article, unless otherwise stated. 


\section{Background}

Successful recruitment and retention are critical for evaluating intervention effectiveness in clinical trials that address childhood obesity. However, the recruitment and retention of participants is challenging, especially in clinical trials that involve ethnic minority or low-income populations in the prevention or treatment of childhood obesity. Problems in participant recruitment may lead to untimely delays in implementation, added financial burden, and failure to meet recruitment goals. Once participants have been recruited, maintaining their engagement across the course of the trial requires thoughtful planning, careful monitoring, and sometimes extraordinary efforts.

Recently, the National Heart, Lung, and Blood Institute convened a workshop to address recruitment and retention strategies in phase 3 and 4 clinical trials. In an article about this initiative, Probstfield and Frye [1] summarized critical steps that must be taken to ensure adequate participant enrollment and retention. These authors noted that trials that involve women and minority populations are more challenging and costly because of issues related to transportation, childcare, and individual and community acceptance. Moreover, reaching minority participants creates additional challenges.

Childhood obesity studies, both for prevention and treatment, present additional challenges related to participant recruitment and retention. Parents and caregivers are often not interested in or have little concern for obesity as a problem and may not recognize excess body weight, especially when it occurs in younger children $[2,3]$. An added component of research involving children is that family participation, either direct or indirect, is required. Even when parents or other primary caregivers are not targeted as study participants, family members must provide consent, support, and coordination for the child's participation in the research study. Thus, recruitment and retention of participants must consider the index child and a parent or guardian for study success.

Childhood obesity intervention trials, especially those conducted within community settings, offer great challenges for participant recruitment and retention because of the time required for baseline measures, intervention delivery, post-intervention testing, and measures of sustainability. Although successful recruitment and retention strategies have been generally described in studies focusing on adults [4] and children [3], no prior reviews have systematically assessed the recruitment and retention of minority or low-income children and families in obesity treatment and prevention studies. In addition, no studies have attempted to determine what information about recruitment and retention is provided in childhood obesity intervention studies following their completion.
More information is needed about successful recruitment and retention strategies for interventions that involve minority or low-income children and families to provide researchers with needed information for better design and budgeting for research studies.

The United States Clinical Trials Registration Database (CTRD) offers an excellent study frame to address these issues. For this database, a clinical trial is defined as any research study that assigns human participants to interventions (e.g., a medical product, behavior, or procedure) to evaluate the effects on health outcomes [5]. In 2000, the United States CTRD (ClinicalTrials.gov) was established as an official web platform and catalog for registering a clinical trial. Run by the United States National Library of Medicine, ClinicalTrials.gov was the first online registry for clinical trials and is the largest and most widely used trial registry today. Part of the purpose of the CTRD is to make clinical trial information more widely available and to standardize information provided about trials. In 2005, the International Committee of Medical Journal Editors initiated the policy that trials will be considered for publication only if they were registered before submission [6]. This policy has been followed by a large number of journals [7]. The CTRD is accepted by the International Committee of Medical Journal Editors [6].

Because of the importance of recruitment and retention strategies, the increased participation of community intervention trials in the CTRD, and the provision of information on the trials' process, a review of the recruitment and retention strategies of childhood obesity prevention and treatment intervention studies located within the database was undertaken. The purpose of the review was to glean collective information from the registered trials, which could be used to improve subsequent childhood obesity interventions and to enhance future recruitment and retention efforts. Specifically, this review aimed to (1) describe strategies employed to recruit minority participants to intervention trials targeting child diet, physical activity, or obesity-related outcomes and assess the success of these recruitment efforts; and (2) examine strategies used to retain participants in these intervention trials and evaluate retention success.

\section{Methods}

The CTRD was searched to identify 'completed' trials (as defined by CTRD) that contained information about recruitment and retention of child or adolescent participants in studies with diet, physical activity, or obesityrelated outcomes on 6 March 2014. We included home-, community-, and school-based interventions with a behavioral intervention component. Inclusion criteria included: (a) ethnic minority or low-income children 
or adolescents as the intervention target; (b) diet, physical activity, or obesity-related outcome; (c) a completed trial; and (d) specific information on recruitment or retention numbers and strategies used. Studies were excluded if they tested a specialized diet, medication, dietary supplement, or monitoring device; studied infants (i.e., <2 years of age); or focused on an infectious disease outcome or illness other than obesity or diabetes.

Using the CTRD search engine, specific search terms used included: (underserved OR 'hidden population' OR uninsured OR minority OR low income OR Latino OR Latina OR Hispanic OR black OR African American OR Mexican American OR poverty OR vulnerable OR ethnic). Also within the CTRD search engine: the 'Recruitment' parameter was constrained to be 'completed'; the 'Study type' parameter was constrained to 'interventional studies'; the 'Conditions' parameter was constrained to (type 2 diabetes OR diabetes mellitus OR obesity OR overweight OR diet OR nutrition OR physical activity OR sedentary behavior); and the 'Age group' parameter was constrained to 'Child (birth to 17 years)'.

As secondary sources of information on recruitment and retention, we searched within CTRD for pertinent papers associated with each identified study. In addition, a PubMed search was conducted using the following information: (CTRD number OR grant number OR intervention name noted in the CTRD) AND name of the principal investigator AND date of study start. All searches of the CTRD and PubMed were conducted by the first author $(\mathrm{ZC})$ after consulting a university librarian assigned to services exclusively for public health research. The first author (ZC) read all of the registration information in an effort to identify appropriate studies. Studies that provided information on recruitment or retention numbers and strategies were retained. Data extraction was performed independently by two authors using tailored tables, and results were crosschecked for accuracy and completeness. Disagreements between the two authors were discussed and resolved in regular writing group meetings.

\section{Results}

\section{Analytic sample and sample characteristics}

A total of 98 studies were retrieved from our search of the CTRD (Fig. 1). Of these, 57 studies were excluded for the following reasons: drug trials $(n=10)$; special diet trials $(n=8)$; dietary supplement $(n=18)$; infectious disease $(n=3)$; monitoring device $(n=5)$; 2-day trial $(n=1)$; participants younger than 2 years $(n=9)$ or older than 17 years $(n=3)$. This yielded a total of 41 eligible studies. Search methods identified two additional papers that described studies that were linked to two of the 41 CTRD numbers but appeared to represent slightly different studies (different sample sizes). These were included

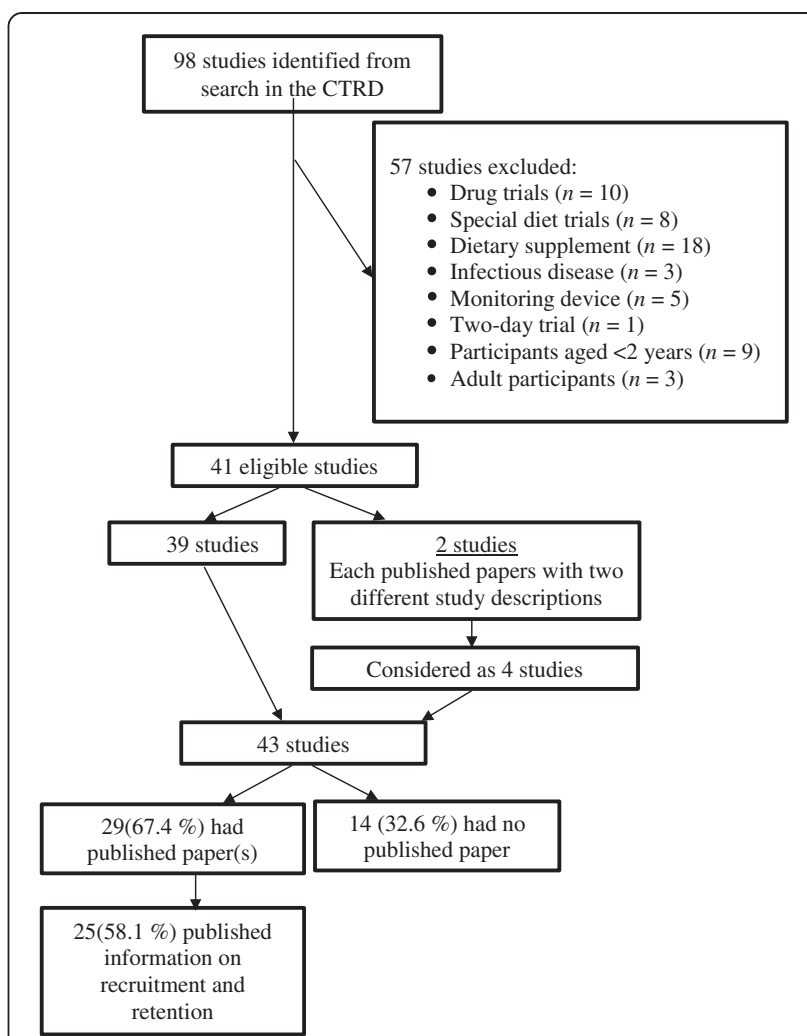

Fig. 1 Flowchart for identification of studies and published papers

as separate studies, bringing our final analytic sample total to 43 studies. Of these 43 studies, 29 had at least one published article in a peer-reviewed journal, with 25 having specific information on recruitment or retention of participants. One of the 25 studies (i.e., Girls Health Enrichment Multi-Site Studies or GEMS) included several articles published, from seven different study phases or sites.

Characteristics of the 25 studies included in this review are described in Table 1 . More than half of the studies were randomized controlled trials $(n=14)$; five were cluster randomized controlled trials; two were nonrandomized controlled trials; and four were trials without a control group. Studies were conducted in various settings, including home or community, including county extension offices, YMCA and childcare centers $(n=11)$, schools $(n=7)$, clinics $(n=5)$, laboratories $(n=3)$. Categories are not mutually exclusive, as some studies had more than one setting. By design, all studies enrolled Hispanics or African Americans, but could have enrolled white participants. Eighty percent of the studies targeted both children and parents. More than $75 \%$ of studies included both nutrition and physical activity intervention components. Two-thirds of the studies lasted less than 1 year. Most studies utilized body mass index (BMI, $n=11$ ) or insulin sensitivity or blood 
Table 1 Characteristics of extracted studies

\begin{tabular}{|c|c|c|c|c|c|c|c|c|}
\hline \multirow{2}{*}{$\begin{array}{l}\text { Reference } \\
\text { and CTRD } \\
\text { number }\end{array}$} & \multicolumn{4}{|l|}{ Participants } & \multicolumn{3}{|l|}{ Intervention } & \multirow[t]{2}{*}{ Primary outcome } \\
\hline & $\begin{array}{l}\text { Child's race or } \\
\text { ethnicity }\end{array}$ & $\begin{array}{l}\text { Child's body } \\
\text { weight } \\
\text { status }\end{array}$ & $\begin{array}{l}\text { Child's age } \\
\text { in years } \\
(\text { sex) }\end{array}$ & $\begin{array}{l}\text { Parental } \\
\text { participation }\end{array}$ & Setting & Focus & Length & \\
\hline $\begin{array}{l}\text { Hasson et al. } \\
\text { [14] }\end{array}$ & \multirow[t]{2}{*}{ Black } & \multirow[t]{2}{*}{ Obese } & \multirow[t]{2}{*}{$15.4 \pm 1.1$} & \multirow[t]{2}{*}{ Yes } & \multirow[t]{2}{*}{ Laboratory } & \multirow{2}{*}{$\begin{array}{l}\text { Nutrition, } \\
\text { physical } \\
\text { activity }\end{array}$} & \multirow[t]{2}{*}{16 weeks } & \multirow[t]{2}{*}{$\begin{array}{l}\text { Adiposity, inflammation } \\
\text { insulin sensitivity }\end{array}$} \\
\hline NCT01441323 & & & & & & & & \\
\hline $\begin{array}{l}\text { Davis et al. } \\
\text { [15] }\end{array}$ & \multirow[t]{3}{*}{ Hispanic } & \multirow[t]{3}{*}{$\begin{array}{l}\text { Overweight } \\
\text { or obese }\end{array}$} & \multirow[t]{3}{*}{$14-18$} & \multirow[t]{3}{*}{ Yes } & \multirow[t]{3}{*}{ Laboratory } & \multirow{3}{*}{$\begin{array}{l}\text { Nutrition, } \\
\text { physical } \\
\text { activity }\end{array}$} & \multirow[t]{3}{*}{16 weeks } & \multirow[t]{3}{*}{$\begin{array}{l}\text { Adiposity, insulin } \\
\text { sensitivity }\end{array}$} \\
\hline $\begin{array}{l}\text { Ventura et al. } \\
{[16]}\end{array}$ & & & & & & & & \\
\hline NCT00697580 & & & & & & & & \\
\hline $\begin{array}{l}\text { Azevedo } \\
\text { et al. [17] }\end{array}$ & \multirow[t]{2}{*}{ Hispanic } & \multirow[t]{2}{*}{ All weights } & \multirow[t]{2}{*}{$7-11$} & \multirow[t]{2}{*}{ Yes } & \multirow{2}{*}{$\begin{array}{l}\text { Not reported } \\
\text { for dance; at } \\
\text { home for TV } \\
\text { time }\end{array}$} & \multirow{2}{*}{$\begin{array}{l}\text { Nutrition, } \\
\text { physical } \\
\text { activity }\end{array}$} & \multirow[t]{2}{*}{2 years } & \multirow[t]{2}{*}{ BMl } \\
\hline NCT00476775 & & & & & & & & \\
\hline $\begin{array}{l}\text { Berry et al. } \\
{[18-20]^{\text {b }}}\end{array}$ & \multirow{2}{*}{$\begin{array}{l}\text { Black (63\%), } \\
\text { white (32\%), } \\
\text { other (5 \%) }\end{array}$} & $\begin{array}{l}\text { Overweight } \\
\text { or obese }\end{array}$ & $7-10$ & $\begin{array}{l}\text { Overweight } \\
\text { or obese }\end{array}$ & School & $\begin{array}{l}\text { Nutrition, } \\
\text { physical }\end{array}$ & 12 months & $\begin{array}{l}\text { Child's BMI percentile, } \\
\text { parent BMI }\end{array}$ \\
\hline NCT01378806 & & & & & & activity & & \\
\hline 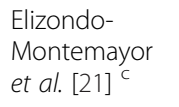 & Hispanic & $\begin{array}{l}\text { Overweight } \\
\text { or obese }\end{array}$ & $6-12$ & Yes & School & Nutrition & 1 school year & $\begin{array}{l}\text { BMI percentile, dietary } \\
\text { intake and eating habits }\end{array}$ \\
\hline NCT01925976 & & & & & & & & \\
\hline $\begin{array}{l}\text { Wang et al. } \\
{[22,23]^{b}}\end{array}$ & Black & All weights & $\begin{array}{l}5-7 \text { th } \\
\text { grade }\end{array}$ & No & School & $\begin{array}{l}\text { Nutrition, } \\
\text { physical }\end{array}$ & & $\begin{array}{l}\text { Feasibility of } \\
\text { intervention }\end{array}$ \\
\hline NCT00061165 & & & & & & & & \\
\hline $\begin{array}{l}\text { Black et al. } \\
{[24,25]}\end{array}$ & Black & All weights & $11-16$ & Yes & $\begin{array}{l}\text { Home and } \\
\text { community }\end{array}$ & $\begin{array}{l}\text { Nutrition, } \\
\text { physical }\end{array}$ & 11 months & $\mathrm{BMI}$ \\
\hline $\begin{array}{l}\text { Hurley et al. } \\
{[26]}\end{array}$ & & & & & & activity & & \\
\hline $\begin{array}{l}\text { Witherspoon } \\
\text { et al. [27] }\end{array}$ & & & & & & & & \\
\hline NCT00746083 & & & & & & & & \\
\hline $\begin{array}{l}\text { Weigensberg } \\
\text { et al. [28] }\end{array}$ & Hispanic & Obese & $14-17$ & No & Not clear & $\begin{array}{l}\text { Nutrition, } \\
\text { physical }\end{array}$ & 12 weeks & Insulin sensitivity \\
\hline NCT01895595 & & & & & & $\begin{array}{l}\text { activity, } \\
\text { interactive } \\
\text { guided } \\
\text { imagery }\end{array}$ & & \\
\hline $\begin{array}{l}\text { Wilson et al. } \\
2011[29-31]^{b}\end{array}$ & $\begin{array}{l}\text { Black (73 \%), } \\
\text { other }\end{array}$ & All weights & 6 th grade & No & School & $\begin{array}{l}\text { Physical } \\
\text { activity }\end{array}$ & 17 weeks & $\begin{array}{l}\text { Moderate-to-vigorous } \\
\text { physical activity }\end{array}$ \\
\hline NCT01028144 & & & & & & & & \\
\hline $\begin{array}{l}\text { Naar-King } \\
\text { et al. [32] }\end{array}$ & Black & Obese & $12-17$ & Yes & Home & $\begin{array}{l}\text { Nutrition, } \\
\text { physical }\end{array}$ & 6 months & $\begin{array}{l}\text { BMI, overweight (\%), } \\
\text { percentage body fat }\end{array}$ \\
\hline NCT00604981 & & & & & & & & \\
\hline $\begin{array}{l}\text { Ritchie et al. } \\
\text { [33] }\end{array}$ & Black & Overweight & $9-10$ & Yes & YMCA & $\begin{array}{l}\text { Nutrition, } \\
\text { physical }\end{array}$ & 4-9 seasons & Insulin sensitivity \\
\hline $\begin{array}{l}\text { Sharma et al. } \\
{[34]^{\mathrm{d}}}\end{array}$ & & & & & & & & \\
\hline NCT01039116 & & & & & & & & \\
\hline $\begin{array}{l}\text { Eisenmann } \\
\text { et al. [35] }\end{array}$ & $\begin{array}{l}\text { Hispanic or } \\
\text { black }\end{array}$ & All weights & $\begin{array}{l}\text { 3rd-5th } \\
\text { grade }\end{array}$ & Yes & $\begin{array}{l}\text { School and } \\
\text { community }\end{array}$ & $\begin{array}{l}\text { Nutrition, } \\
\text { physical }\end{array}$ & 2 years & $\begin{array}{l}\text { Physical activity, healthy } \\
\text { eating index }\end{array}$ \\
\hline NCT01385046 & & & & & & activity & & \\
\hline & Hispanic & All weights & $2-6$ & Yes & & & 12 weeks & $\mathrm{BMI}$ \\
\hline
\end{tabular}


Table 1 Characteristics of extracted studies (Continued)

\begin{tabular}{|c|c|c|c|c|c|c|c|c|}
\hline $\begin{array}{l}\text { Barkin et al. } \\
\text { [36] } \\
\text { NCT00808431 }\end{array}$ & & & & & $\begin{array}{l}\text { Community } \\
\text { recreation } \\
\text { center }\end{array}$ & $\begin{array}{l}\text { Nutrition, } \\
\text { physical } \\
\text { activity }\end{array}$ & & \\
\hline $\begin{array}{l}\text { Burnet et al. } \\
{[37]^{e}} \\
\text { NCT00723853 }\end{array}$ & Black & $\begin{array}{l}\text { Overweight } \\
\text { or obese }\end{array}$ & $\begin{array}{l}\text { 9-12, with } \\
\text { family } \\
\text { history of } \\
\text { type } 2 \\
\text { diabetes } \\
\text { mellitus }\end{array}$ & Yes & Community & $\begin{array}{l}\text { Nutrition, } \\
\text { physical } \\
\text { activity }\end{array}$ & 1 year & $\begin{array}{l}\text { Child's BMI z score, } \\
\text { parent's BMI, glucose } \\
\text { tolerance }\end{array}$ \\
\hline $\begin{array}{l}\text { Davis et al. } \\
{[38-40]} \\
\text { Tkacz et al. } \\
{[41]}\end{array}$ & $\begin{array}{l}\text { White (58\%), } \\
\text { black (39\%), } \\
\text { Hispanic (3\%) }\end{array}$ & $\begin{array}{l}\text { Overweight } \\
\text { or obese }\end{array}$ & $7-11$ & No & Laboratory & $\begin{array}{l}\text { Nutrition, } \\
\text { physical } \\
\text { activity }\end{array}$ & 10-15 weeks & $\begin{array}{l}\text { Risk of type } 2 \text { diabetes } \\
\text { mellitus, } \mathrm{VO}_{2} \text { max, } \\
\text { percentage body fat, } \\
\text { visceral fat }\end{array}$ \\
\hline $\begin{array}{l}\text { Petty et al. } \\
\text { [42] } \\
\text { NCT00108901 }\end{array}$ & & & & & & & & \\
\hline $\begin{array}{l}\text { Madsen et al. } \\
\text { [43] }^{\text {b }} \\
\text { NCT01156103 }\end{array}$ & $\begin{array}{l}\text { Hispanic (42\%), } \\
\text { Asian (32\%), } \\
\text { black (12\%), } \\
\text { white }(0.6 \%), \\
\text { other (13.4\%) }\end{array}$ & All weights & $\begin{array}{l}4 \text { th or } 5 \text { th } \\
\text { grade }\end{array}$ & No & School & $\begin{array}{l}\text { Physical } \\
\text { activity }\end{array}$ & 24 weeks & $\begin{array}{l}\text { Change in minutes of } \\
\text { after-school moderate- } \\
\text { to-vigorous physical ac- } \\
\text { tivity, } \mathrm{VO}_{2} \text { max, BMI }\end{array}$ \\
\hline $\begin{array}{l}\text { Wickham } \\
\text { et al. [44] } \\
\text { NCT00167830 }\end{array}$ & $\begin{array}{l}\text { Black (70.3\%), } \\
\text { white (26.1\%), } \\
\text { Hispanic (1.8\%) }\end{array}$ & Obese & $11-18$ & Yes & $\begin{array}{l}\text { Weight } \\
\text { management } \\
\text { clinic }\end{array}$ & $\begin{array}{l}\text { Nutrition, } \\
\text { physical } \\
\text { activity }\end{array}$ & $\begin{array}{l}2 \text { years (results } \\
\text { at } 6 \text { months } \\
\text { reported) }\end{array}$ & $\begin{array}{l}\text { BMI, metabolic } \\
\text { indicators, fitness }\end{array}$ \\
\hline $\begin{array}{l}\text { Bean et al. } \\
{[45]^{\mathrm{e}}} \\
\text { NCT00167830 }\end{array}$ & $\begin{array}{l}\text { Black (75.3\%), } \\
\text { white }(22.0 \%) \text {, } \\
\text { other }(2.7 \%)\end{array}$ & Obese & $11-18$ & Yes & $\begin{array}{l}\text { Weight } \\
\text { management } \\
\text { clinic }\end{array}$ & $\begin{array}{l}\text { Nutrition, } \\
\text { physical } \\
\text { activity }\end{array}$ & $\begin{array}{l}2 \text { years (results } \\
\text { at } 6 \text { months } \\
\text { reported) }\end{array}$ & Dietary changes \\
\hline $\begin{array}{l}\text { Wysocki et al. } \\
{[46,47]} \\
\text { NCT00358059 }\end{array}$ & $\begin{array}{l}\text { White }(78.2 \%) \text {, } \\
\text { black (21.0\%), } \\
\text { Hispanic }(0.8 \%)\end{array}$ & All weights & $\begin{array}{l}\text { 12-16.75 } \\
\text { with type } 1 \\
\text { diabetes } \\
\text { mellitus }\end{array}$ & Yes & $\begin{array}{l}\text { Treatment } \\
\text { center }\end{array}$ & $\begin{array}{l}\text { Parent- } \\
\text { adolescent } \\
\text { conflict }\end{array}$ & $\begin{array}{l}12 \text { months } \\
\text { (results at } \\
3 \text { months } \\
\text { reported) }\end{array}$ & $\begin{array}{l}\text { Family relationships, } \\
\text { psychological } \\
\text { adjustment to diabetes } \\
\text { treatment, treatment } \\
\text { adherence, diabetic } \\
\text { control }\end{array}$ \\
\hline $\begin{array}{l}\text { Wysocki et al. } \\
\text { [48-50] } \\
\text { NCT00358059 }\end{array}$ & $\begin{array}{l}\text { White }(63.5 \%) \text {, } \\
\text { black }(30.8 \%) \text {, } \\
\text { Hispanic } \\
(2.9 \%) \text {, other } \\
(2.9 \%)\end{array}$ & All weights & $\begin{array}{l}\text { 11-16, } \\
\text { with type } 1 \\
\text { diabetes } \\
\text { mellitus }\end{array}$ & Yes & $\begin{array}{l}\text { Pediatric } \\
\text { center }\end{array}$ & $\begin{array}{l}\text { Parent- } \\
\text { adolescent } \\
\text { conflict }\end{array}$ & 6 months & $\begin{array}{l}\text { Family relationships, } \\
\text { treatment adherence, } \\
\mathrm{HbA1c} \text {, health care use }\end{array}$ \\
\hline $\begin{array}{l}\text { Ellis et al. [51, } \\
\text { 52] } \\
\text { NCT00519935 }\end{array}$ & $\begin{array}{l}\text { Black }(63 \%), \\
\text { white }(26 \%), \\
\text { Other }(11 \%)\end{array}$ & All weights & $\begin{array}{l}\text { 10-17, } \\
\text { with type } 1 \\
\text { diabetes } \\
\text { mellitus }\end{array}$ & Yes & $\begin{array}{l}\text { Home, } \\
\text { community }\end{array}$ & $\begin{array}{l}\text { Home-based } \\
\text { psychotherapy }\end{array}$ & $\begin{array}{l}\text { Approximately } \\
6 \text { months }\end{array}$ & $\begin{array}{l}\text { Adherence to medical } \\
\text { regimen, metabolic } \\
\text { control, hospital use }\end{array}$ \\
\hline Story et al. [2] & Black & $\begin{array}{l}\text { Phase I: BMI } \\
\geq 25 \text { th or } \\
\geq 50 \text { th } \\
\text { percentile; }\end{array}$ & 8-10 (girls) & $\begin{array}{l}\text { Overweight } \\
\text { or obese }\end{array}$ & $\begin{array}{l}\text { Community } \\
\text { center, school, } \\
\text { home }\end{array}$ & $\begin{array}{l}\text { Nutrition, } \\
\text { physical } \\
\text { activity }\end{array}$ & $\begin{array}{l}\text { Phase I: } \\
12 \text { weeks; }\end{array}$ & $\begin{array}{l}\text { Phase I: process } \\
\text { measures; }\end{array}$ \\
\hline $\begin{array}{l}\text { Rochon et al. } \\
{[53]} \\
\text { Kumanyika } \\
\text { et al. }[54,55]\end{array}$ & & $\begin{array}{l}\text { Phase II: } \\
\text { BMI } \geq 25 \text { th } \\
\text { percentile } \\
\text { but } \leq 35 \mathrm{~kg} / \\
\mathrm{m}^{2}\end{array}$ & & & & & $\begin{array}{l}\text { Phase } \| \text { : } \\
2 \text { years }\end{array}$ & $\begin{array}{l}\text { Phase II: change in } \\
\text { child's BMI }\end{array}$ \\
\hline
\end{tabular}

Klesges et al.

$[56,57]$

Robinson

et al. $[58,59]$

Stockton

et al. [60]

NCT00000615 
Table 1 Characteristics of extracted studies (Continued)

\begin{tabular}{|c|c|c|c|c|c|c|c|c|}
\hline $\begin{array}{l}\text { Natale et al. } \\
\text { [61] }^{\text {b }} \\
\text { NCT01722032 }\end{array}$ & $\begin{array}{l}\text { Hispanic (60\%), } \\
\text { Haitian (15\%), } \\
\text { black (12\%), } \\
\text { white (2\%), } \\
\text { other (11\%) }\end{array}$ & All weights & $2-5$ & Yes & $\begin{array}{l}\text { Childcare } \\
\text { center }\end{array}$ & $\begin{array}{l}\text { Nutrition, } \\
\text { physical } \\
\text { activity }\end{array}$ & 2 years & Child's BMl \\
\hline $\begin{array}{l}\text { Nansel et al. } \\
{[62]} \\
\text { NCT00273286 }\end{array}$ & $\begin{array}{l}\text { White }(75 \%), \\
\text { Hispanic (10\%), } \\
\text { black }(9 \%), \\
\text { other }(6 \%)\end{array}$ & All weights & $\begin{array}{l}\text { 9-14.9, } \\
\text { with type } 1 \\
\text { diabetes } \\
\text { mellitus }\end{array}$ & Yes & $\begin{array}{l}\text { Pediatric } \\
\text { endocrinology } \\
\text { clinic }\end{array}$ & $\begin{array}{l}\text { Diabetes } \\
\text { management } \\
\text { behavior }\end{array}$ & 2 years & $\mathrm{HbA} 1 \mathrm{c}$ \\
\hline $\begin{array}{l}\text { Janicke et al. } \\
\text { [63] } \\
\text { Follansbee- } \\
\text { Junger et al. } \\
\text { [64] }\end{array}$ & \multirow[t]{3}{*}{$\begin{array}{l}\text { White }(76.1 \%), \\
\text { black }(9.8 \%), \\
\text { Hispanic } \\
(8.5 \%), \text { biracial } \\
(4.2 \%)\end{array}$} & \multirow[t]{3}{*}{$\begin{array}{l}\text { Overweight } \\
\text { or obese }\end{array}$} & \multirow[t]{3}{*}{$8-14$} & \multirow[t]{3}{*}{ Yes } & \multirow[t]{3}{*}{$\begin{array}{l}\text { County } \\
\text { extension } \\
\text { office }\end{array}$} & \multirow[t]{3}{*}{$\begin{array}{l}\text { Nutrition, } \\
\text { physical } \\
\text { activity }\end{array}$} & \multirow[t]{3}{*}{16 weeks } & \multirow[t]{3}{*}{ Change in child's BMI } \\
\hline $\begin{array}{l}\text { Radcliff et al. } \\
\text { [65] }\end{array}$ & & & & & & & & \\
\hline NCT00248677 & & & & & & & & \\
\hline
\end{tabular}

$B M I$ body mass index

ancluded both sexes if not specified

${ }^{\mathrm{b}}$ Cluster randomized clinical trial

'Trial without control group

${ }^{\mathrm{d}}$ Non-randomized controlled trial

epre-post study design

glucose metabolism $(n=10)$ as the primary outcome measures, while others used physical activity or fitness $(n=5)$, body fat $(n=4)$, diet $(n=3)$ or adherence behaviors $(n=3)$.

\section{Recruitment rates and strategies}

Recruitment information provided in the studies is described in Table 2. Of the 25 studies, 16 (64\%) did not report a recruitment target; 8 (32\%) did not report capture rate expressed as the ratio of participants who were enrolled to potential participants who were screened. When capture rate was included, it ranged from $10 \%$ to $90 \%$. Eight (32\%) of the 25 studies did not report formative research information on recruitment. Only eight studies reported recruitment durations, which ranged from 2.5 months (enrolled approximately 60 girls) to 3 years (enrolled 235 children). Recruitment was primarily conducted in community, school, and primary care settings. Specific recruitment strategies were reported in only 14 studies, with the amount of information varying from a single comment to several pages. Common recruitment methods were presentations, flyers, brochures, posters, media advertisements, phone calls, and word-of-mouth. Two-thirds of studies did not report any information on barriers for recruitment. When barriers were reported, they included participants' time constraints, competing demands, transportation safety and distance, childcare needs, lack of interest, and study funding limitations.

\section{Retention rates and strategies}

Table 3 shows the average retention rates from individual studies based on study characteristics. Of the 25 studies examined, 23 studies reported retention rates, with an average rate of $86 \%$. Studies solely targeting Hispanics or African Americans had lower average retention rates, of $82.8 \%$ and $83.5 \%$, respectively, than those targeting both ethnic minority and white participants (92.1\%). Three studies included children only; the average retention rate from these studies was higher than the average retention rate from studies that involved both children and parents (91.1\% vs. $85.6 \%$ ). On average, studies that focused on overweight or obese children had lower retention rates than those that targeted children generally (79.6 \% vs. $90.0 \%$ ). Accordingly, treatment studies had a lower average retention rate than prevention studies, especially when the intervention lasted over 1 year (74.0 \% vs. $88.8 \%$ ). Overall, longer-term studies produced lower retention rates than shorter-term studies, especially for treatment studies $(74.0 \%$ for $\geq 1$ year vs. $87.2 \%$ for $<1$ year). Interestingly, studies with BMI or anthropometrics as primary outcome measures had lower retention rates than studies with other primary outcome measures (e.g., obesity-related behavior, insulin sensitivity; 82.9 \% vs. $89.0 \%$ ). Home- or community-based studies had lower retention rates than school-based studies (85.5 \% vs. $91.7 \%)$. Studies including both nutrition and physical activity intervention components tended to have lower retention rates than studies focusing solely on nutrition or physical activity ( $85.0 \%$ vs. $92.8 \%$ ). Retention rates did not differ by the mean age of children $(<12$ years vs. $\geq$ 12 years), number of intervention sessions ( $\leq 12$ vs. $\geq 13$ ), or study sample size ( $<100$ vs. $\geq 100)$.

Of the 25 studies, 18 (72\%) reported retention strategies. We analyzed and coded retention strategies used 
Table 2 Study recruitment: effectiveness, setting, strategies employed, and barriers reported

\begin{tabular}{|c|c|c|c|c|c|c|c|}
\hline Reference & $\begin{array}{l}\text { Sample } \\
\text { size }\end{array}$ & $\begin{array}{l}\text { Reach } \\
\text { (\% } \\
\text { capture) }\end{array}$ & $\begin{array}{l}\text { Formative } \\
\text { research }\end{array}$ & $\begin{array}{l}\text { Recruitment } \\
\text { duration }\end{array}$ & Recruitment setting & $\begin{array}{l}\text { Recruitment } \\
\text { strategies }\end{array}$ & Recruitment barriers \\
\hline $\begin{array}{l}\text { Hasson et al. } \\
{[14]}\end{array}$ & 58 families & 11.6 & Yes & - & - & - & - \\
\hline $\begin{array}{l}\text { Davis et al. } \\
\text { [15] }\end{array}$ & 68 families & 17.0 & Yes & - & - & - & - \\
\hline \multicolumn{8}{|l|}{$\begin{array}{l}\text { Ventura et al. } \\
{[16]}\end{array}$} \\
\hline $\begin{array}{l}\text { Azevedo } \\
\text { et al. [17] }\end{array}$ & $\begin{array}{l}252 \\
\text { families }\end{array}$ & - & - & - & Community & - & - \\
\hline \multirow[t]{5}{*}{$\begin{array}{l}\text { Berry et al. } \\
{[18-20]}\end{array}$} & \multirow{5}{*}{$\begin{array}{l}358 \\
\text { parent- } \\
\text { child dyads }\end{array}$} & \multirow[t]{5}{*}{27.5} & \multirow[t]{5}{*}{ Yes } & \multirow[t]{5}{*}{$\begin{array}{l}2 \text { years } \\
9 \text { months }\end{array}$} & \multirow[t]{5}{*}{ School } & $\begin{array}{l}\text { 1) Meeting with } \\
\text { school staff }\end{array}$ & \multirow[t]{5}{*}{-} \\
\hline & & & & & & $\begin{array}{l}\text { 2) Printed study } \\
\text { information }\end{array}$ & \\
\hline & & & & & & $\begin{array}{l}\text { 3) Presentation to } \\
\text { children and parents }\end{array}$ & \\
\hline & & & & & & $\begin{array}{l}\text { 4) Printed study } \\
\text { contact information }\end{array}$ & \\
\hline & & & & & & 5) Friendly manner & \\
\hline $\begin{array}{l}\text { Elizondo- } \\
\text { Montemayor } \\
\text { et al. [21] }\end{array}$ & $\begin{array}{l}125 \\
\text { caregiver- } \\
\text { child dyads }\end{array}$ & 9.6 & - & - & School & - & - \\
\hline $\begin{array}{l}\text { Wang et al. } \\
{[22,23]}\end{array}$ & $\begin{array}{l}249 \\
\text { children }\end{array}$ & 37.1 & Yes & - & School & - & - \\
\hline $\begin{array}{l}\text { Black et al. } \\
{[24,25]}\end{array}$ & $\begin{array}{l}235 \\
\text { children }\end{array}$ & - & - & $\begin{array}{l}1 \text { year } \\
10 \text { months }\end{array}$ & School & - & - \\
\hline \multicolumn{8}{|l|}{$\begin{array}{l}\text { Hurley et al. } \\
{[26]}\end{array}$} \\
\hline \multicolumn{8}{|l|}{$\begin{array}{l}\text { Witherspoon } \\
\text { et al. [27] }\end{array}$} \\
\hline $\begin{array}{l}\text { Weigensberg } \\
\text { et al. [28] }\end{array}$ & $\begin{array}{l}35 \\
\text { adolescents }\end{array}$ & 62.5 & Yes & - & Pediatric clinics, health fairs & - & School vacation \\
\hline \multirow[t]{2}{*}{$\begin{array}{l}\text { Wilson et al. } \\
2011 \text { [29-31] }\end{array}$} & \multirow[t]{2}{*}{$\begin{array}{l}1422 \\
\text { children }\end{array}$} & \multirow[t]{2}{*}{91.0} & \multirow[t]{2}{*}{ Yes } & \multirow[t]{2}{*}{-} & \multirow[t]{2}{*}{ School and home } & $\begin{array}{l}\text { 1) Presentation to } \\
\text { parents and } \\
\text { students }\end{array}$ & \multirow[t]{2}{*}{-} \\
\hline & & & & & & 2) Home visit & \\
\hline \multirow{2}{*}{$\begin{array}{l}\text { Naar-King } \\
\text { et al. [32] }\end{array}$} & \multirow[t]{2}{*}{49 families } & \multirow[t]{2}{*}{69.0} & \multirow[t]{2}{*}{ Yes } & \multirow[t]{2}{*}{-} & \multirow{2}{*}{$\begin{array}{l}\text { An urban adolescent } \\
\text { medicine clinic }\end{array}$} & \multirow[t]{2}{*}{-} & 1) Time constraint; \\
\hline & & & & & & & 2) Lack of interest \\
\hline $\begin{array}{l}\text { Ritchie et al. } \\
\text { [33] }\end{array}$ & $\begin{array}{l}235 \\
\text { families }\end{array}$ & - & Yes & 3 years & School, community & 1) Announcements & 1) Transportation; \\
\hline \multirow{2}{*}{$\begin{array}{l}\text { Sharma et al. } \\
\text { [34] }\end{array}$} & & & & & & 2) Incentives & 2) Competing demands; \\
\hline & & & & & & & 3) Distrust; \\
\hline $\begin{array}{l}\text { Eisenmann } \\
\text { et al. [35] }\end{array}$ & $\begin{array}{l}434 \\
\text { families }\end{array}$ & 57.0 & - & - & School & - & - \\
\hline \multirow[t]{3}{*}{$\begin{array}{l}\text { Barkin et al. } \\
{[36]}\end{array}$} & \multirow{3}{*}{$\begin{array}{l}106 \\
\text { parent- } \\
\text { child dyads }\end{array}$} & \multirow[t]{3}{*}{22.2} & \multirow[t]{3}{*}{-} & \multirow[t]{3}{*}{ 4-5 months } & \multirow{3}{*}{$\begin{array}{l}\text { Cooperating community } \\
\text { agencies such as social } \\
\text { service agencies, pediatric } \\
\text { clinics, community centers }\end{array}$} & $\begin{array}{l}\text { 1) Printed study } \\
\text { information }\end{array}$ & 1) Transportation; \\
\hline & & & & & & 2) Radio & 2) On-site childcare \\
\hline & & & & & & 3) Participant referral & \\
\hline $\begin{array}{l}\text { Burnet et al. } \\
{[37]}\end{array}$ & 29 families & - & Yes & - & $\begin{array}{l}\text { Community, pediatric } \\
\text { clinics }\end{array}$ & $\begin{array}{l}\text { Printed study } \\
\text { information }\end{array}$ & - \\
\hline $\begin{array}{l}\text { Davis et al. } \\
\text { [38-40] }\end{array}$ & $\begin{array}{l}222 \\
\text { children }\end{array}$ & $26.4 \%$ & - & $\begin{array}{l}2 \text { years } \\
8 \text { months }\end{array}$ & School & $\begin{array}{l}\text { Printed study } \\
\text { information }\end{array}$ & - \\
\hline
\end{tabular}


Table 2 Study recruitment: effectiveness, setting, strategies employed, and barriers reported (Continued)

\begin{tabular}{|c|c|c|c|c|c|c|c|}
\hline \multicolumn{8}{|l|}{$\begin{array}{l}\text { Tkacz et al. } \\
\text { [41] }\end{array}$} \\
\hline \multicolumn{8}{|l|}{$\begin{array}{l}\text { Petty et al. } \\
\text { [42] }\end{array}$} \\
\hline $\begin{array}{l}\text { Madsen et al. } \\
\text { [43] }\end{array}$ & $\begin{array}{l}156 \\
\text { children, } \\
\text { six schools }\end{array}$ & $\begin{array}{l}11.7 \% \\
50 \% \\
89.7 \%\end{array}$ & Yes & - & School & $\begin{array}{l}\text { Presentation to } \\
\text { school staff }\end{array}$ & $\begin{array}{l}\text { Change in school } \\
\text { administration }\end{array}$ \\
\hline $\begin{array}{l}\text { Wickham } \\
\text { et al. [44] }\end{array}$ & $\begin{array}{l}165 \\
\text { adolescents }\end{array}$ & - & - & $\begin{array}{l}2 \text { years } \\
4 \text { months }\end{array}$ & $\begin{array}{l}\text { Comprehensive weight } \\
\text { management program }\end{array}$ & $\begin{array}{l}\text { Healthcare provider } \\
\text { referral }\end{array}$ & - \\
\hline $\begin{array}{l}\text { Bean et al. } \\
{[45]}\end{array}$ & $\begin{array}{l}186 \\
\text { adolescents }\end{array}$ & - & Yes & $\begin{array}{l}2 \text { years } \\
11 \text { months }\end{array}$ & $\begin{array}{l}\text { Health care, school, } \\
\text { community }\end{array}$ & $\begin{array}{l}\text { Healthcare provider } \\
\text { referral }\end{array}$ & - \\
\hline \multirow{2}{*}{$\begin{array}{l}\text { Wysocki et al. } \\
{[46,47]}\end{array}$} & \multirow{2}{*}{$\begin{array}{l}119 \\
\text { families }\end{array}$} & \multirow[t]{2}{*}{$31.3 \%$} & \multirow[t]{2}{*}{ Yes } & \multirow[t]{2}{*}{-} & \multirow[t]{2}{*}{-} & \multirow[t]{2}{*}{-} & 1) Transportation; \\
\hline & & & & & & & 2) Time constraint \\
\hline \multirow[t]{2}{*}{$\begin{array}{l}\text { Wysocki et al. } \\
\text { [48-50] }\end{array}$} & \multirow[t]{2}{*}{$\begin{array}{l}104 \\
\text { families }\end{array}$} & \multirow[t]{2}{*}{$23.9 \%$} & \multirow[t]{2}{*}{ Yes } & \multirow[t]{2}{*}{-} & \multirow[t]{2}{*}{ Pediatric diabetes centers } & $\begin{array}{l}\text { 1) Mailed invitation } \\
\text { letter }\end{array}$ & \multirow[t]{2}{*}{ Funding limitation } \\
\hline & & & & & & 2) Phone call & \\
\hline $\begin{array}{l}\text { Ellis et al. [51, } \\
\text { 52] }\end{array}$ & $\begin{array}{l}127 \\
\text { adolescents }\end{array}$ & $69.8 \%$ & Yes & - & Endocrinology clinic & - & - \\
\hline Story et al. [2] & $\begin{array}{l}\text { Phase I: } \\
\text { 35-61 girls; }\end{array}$ & $\begin{array}{l}\text { Phase I: } \\
\text { not } \\
\text { reported; }\end{array}$ & Yes & $\begin{array}{l}\text { Phase l: } 2.5- \\
4 \text { months; }\end{array}$ & \multirow[t]{8}{*}{$\begin{array}{l}\text { Community churches, } \\
\text { community centers, } \\
\text { community events and } \\
\text { school }\end{array}$} & $\begin{array}{l}\text { 1) Active placebo } \\
\text { study group }\end{array}$ & Phase I: \\
\hline $\begin{array}{l}\text { Rochon et al. } \\
\text { [53] }\end{array}$ & \multirow{7}{*}{$\begin{array}{l}\text { Phase II: } \\
\text { 261-303 } \\
\text { girls }\end{array}$} & \multirow{2}{*}{$\begin{array}{l}\text { Phase II: } \\
48.1 \%{ }^{-} \\
65.4 \%\end{array}$} & & \multirow[t]{2}{*}{$\begin{array}{l}\text { Phase II: } \\
17 \text { months }\end{array}$} & & $\begin{array}{l}\text { 2) Media adverts, } \\
\text { stories, interviews }\end{array}$ & $\begin{array}{l}\text { 1) No-treatment control } \\
\text { group; }\end{array}$ \\
\hline $\begin{array}{l}\text { Kumanyika } \\
\text { et al. }[54,55]\end{array}$ & & & & & & 3) Flyers to homes & $\begin{array}{l}\text { 2) Parents interested in both } \\
\text { child health and self-esteem } \\
\text { programs, while children in- } \\
\text { terested in fun programs; }\end{array}$ \\
\hline $\begin{array}{l}\text { Klesges et al. } \\
{[56,57]}\end{array}$ & & & & & & $\begin{array}{l}\text { 4) Presentations to } \\
\text { families at } \\
\text { community and } \\
\text { school }\end{array}$ & 3) Blood draw. \\
\hline $\begin{array}{l}\text { Robinson } \\
\text { et al. }[58,59]\end{array}$ & & & & & & \multirow{4}{*}{$\begin{array}{l}\text { 5) Separate consent } \\
\text { for blood draw, } \\
\text { which was not } \\
\text { required for } \\
\text { participation }\end{array}$} & Phase II: \\
\hline \multirow{3}{*}{$\begin{array}{l}\text { Stockton } \\
\text { et al. [60] }\end{array}$} & & & & & & & 1) School vacation \\
\hline & & & & & & & 2) Study staff issues \\
\hline & & & & & & & 3) Study site locations \\
\hline $\begin{array}{l}\text { Natale et al. } \\
\text { [61] }\end{array}$ & $\begin{array}{l}1105 \\
\text { children }\end{array}$ & - & - & - & Child care center & - & - \\
\hline $\begin{array}{l}\text { Nansel et al. } \\
{[62]}\end{array}$ & $\begin{array}{l}390 \\
\text { families }\end{array}$ & $69.1 \%$ & - & & $\begin{array}{l}\text { Pediatric endocrinology } \\
\text { clinics }\end{array}$ & - & - \\
\hline $\begin{array}{l}\text { Janicke et al. } \\
\text { [63] }\end{array}$ & $\begin{array}{l}93 \text { parent- } \\
\text { child dyads }\end{array}$ & $83.8 \%$ & Yes & & Community and school & $\begin{array}{l}\text { 1) Printed study } \\
\text { information }\end{array}$ & - \\
\hline $\begin{array}{l}\text { Follansbee- } \\
\text { Junger et al. } \\
\text { [64] }\end{array}$ & & & & & & $\begin{array}{l}\text { 2) Community } \\
\text { presentations }\end{array}$ & \\
\hline $\begin{array}{l}\text { Radcliff et al. } \\
{[65]}\end{array}$ & & & & & & 3) Toll-free line & \\
\hline
\end{tabular}

in these studies and categorized strategies into intervention design, incentive, project bond, participant convenience, and participant tracking (Table 4). Retention strategies related to intervention design included culturally appropriate intervention activities and staff, developmentally appropriate goals for participants, a run-in phase before randomization, provision of counseling or technical support to help participants address participation barriers, regular interventionist-principal investigator meetings to ensure participant-centered intervention, and the use of a delayed or alternative intervention for control group. Incentives, such as grocery gift 
Table 3 Average retention rates by study characteristics

\begin{tabular}{|c|c|c|c|c|}
\hline & Number of studies & Study enrollment ${ }^{a}$ & Study retention ${ }^{\mathrm{b}}$ & Average retention rates \\
\hline \multicolumn{5}{|l|}{ Race or ethnicity } \\
\hline Hispanic & 5 & 586 & 511 & 82.8 \\
\hline African American & 10 & 1331 & 1059 & 83.5 \\
\hline African American, white and other & 8 & 1927 & 1763 & 92.1 \\
\hline \multicolumn{5}{|l|}{ Intervention target } \\
\hline Children & 3 & 413 & 388 & 91.1 \\
\hline Children and parent & 20 & 3431 & 2945 & 85.6 \\
\hline \multicolumn{5}{|l|}{ Body weight status } \\
\hline Overweight or obese & 9 & 1581 & 1314 & 79.6 \\
\hline All weights & 10 & 1523 & 1334 & 90.0 \\
\hline Body weight status not measured & 4 & 740 & 685 & 92.6 \\
\hline \multicolumn{5}{|l|}{ Study type } \\
\hline Prevention & 10 & 1523 & 1334 & 90.0 \\
\hline Treatment & 13 & 2321 & 1999 & 83.6 \\
\hline \multicolumn{5}{|l|}{ Intervention length } \\
\hline$<1$ year & 16 & 1658 & 1461 & 88.6 \\
\hline$\geq 1$ year & 7 & 2186 & 1872 & 81.1 \\
\hline \multicolumn{5}{|l|}{ Study type and treatment length } \\
\hline Prevention $<1$ year & 7 & 707 & 614 & 90.4 \\
\hline Prevention $\geq 1$ year & 3 & 816 & 720 & 88.8 \\
\hline Treatment $<1$ year & 9 & 951 & 847 & 87.2 \\
\hline Treatment $\geq 1$ year & 4 & 1073 & 873 & 74.0 \\
\hline \multicolumn{5}{|l|}{ Primary outcome } \\
\hline BMI or anthropometrics & 10 & 2342 & 2026 & 82.9 \\
\hline Other (behavior, physiology, etc.) & 13 & 1502 & 1307 & 89.0 \\
\hline \multicolumn{5}{|l|}{ Intervention setting ${ }^{c}$} \\
\hline School & 5 & 1273 & 1151 & 91.7 \\
\hline Home or community & 15 & 2410 & 2051 & 85.5 \\
\hline Laboratory & 2 & 126 & 102 & 81.1 \\
\hline \multicolumn{5}{|l|}{ Main intervention group } \\
\hline Nutrition or physical activity & 4 & 755 & 712 & 92.8 \\
\hline Nutrition and physical activity & 19 & 3089 & 2621 & 85.0 \\
\hline \multicolumn{5}{|l|}{ Study design } \\
\hline Randomized controlled trial & 19 & 2739 & 2440 & 89.3 \\
\hline Cluster randomized controlled trial & 2 & 745 & 656 & 75.6 \\
\hline Controlled trial & 1 & 235 & 136 & 57.9 \\
\hline Trial without control & 1 & 125 & 101 & 80.8 \\
\hline \multicolumn{5}{|l|}{ Mean age of children ${ }^{d}$} \\
\hline$<12$ years & 15 & 2708 & 2333 & 86.2 \\
\hline$\geq 12$ years & 8 & 1136 & 1000 & 86.7 \\
\hline \multicolumn{5}{|l|}{ Number of intervention sessions ${ }^{\mathrm{e}}$} \\
\hline$\leq 12$ & 7 & 752 & 636 & 86.3 \\
\hline$\geq 13$ & 15 & 2840 & 2445 & 85.5 \\
\hline
\end{tabular}


Table 3 Average retention rates by study characteristics (Continued)

\begin{tabular}{|c|c|c|c|c|}
\hline \multicolumn{5}{|c|}{ Sample size } \\
\hline$<100$ & 9 & 493 & 435 & 86.9 \\
\hline$\geq 100$ & 14 & 3351 & 2898 & 86.0 \\
\hline
\end{tabular}

cards, gifts, cash, food, recipe books, and exercise equipment, were offered for intervention attendance or completion at each data collection point. Study staff also established project bonds with participants or the broader community by building staff-participant relationships, and regular communication with participants, such as thank-you notes, postcards, or project newsletters. Retention strategies related to participant convenience included transportation support to and from intervention activities or data collection, makeup sessions for missed intervention sessions, upcoming event reminders, childcare services, and optional days or home visits for data collection. To facilitate tracking participants, complete contact information was collected from participants at baseline and a tracking database established. One study mentioned sending personalized letters to participants who were difficult to reach, to schedule data collection appointments. Common retention methods used were alternative or delayed interventions for the control groups, monetary incentives, regular contact and relationship building with participants and the community, provision of transportation support, and offering flexible intervention and measurement visits.

\section{Discussion}

\section{Summary of key findings}

Our systematic review of recruitment and retention of minority or low-income children into obesity-related intervention trials identified 41 completed studies in the CTRD, two of which were linked to two studies. Of these 43 studies, only 25 (60 \%) had published information on recruitment or retention in a peerreviewed article, with considerable variation in the amount of information provided among studies. A further $\approx 10 \%$ included no information about recruitment and retention in their papers. Even when we examined only the studies completed 2 years prior to the close date of our CTRD search, more than $30 \%$ had no publications in peer-review journals. Although most studies with relevant information reported high retention rates, differences in retention rates existed by participant characteristics (i.e., race, obesity status, involving parents or caregivers) and study design (i.e., prevention or treatment, study duration, primary outcome, home-, community-, or school-based).

\section{Previous studies that have examined recruitment and retention in this population}

Two other studies have systematically examined published articles about recruitment and retention of children into obesity-related studies. Schoeppe et al. [3] summarized strategies used to recruit and retain children in behavioral health risk factor studies that achieved high capture rates and low attrition rates, while Amon et al. [8] systematically reviewed literature that included the use of Facebook to recruit 10-18-year-old children into studies that aimed to address physical or mental health issues. The authors found that paid advertising on Facebook was effective in recruiting these participants. These two studies used published literature only as their study frame; thus, their results did not cover studies without publications and could not evaluate the proportion of studies conducted with published information on recruitment and retention. Furthermore, these reviews focused on youth generally; thus, it is unclear whether findings can be generalized to minority or low-income children.

\section{Qualitative and quantitative evidence in recruitment and retention}

The articles identified in our review mainly provided narrative descriptions of recruitment and retention strategies used, investigators' opinions on the effectiveness of these strategies, and lessons learned in individual studies. While this describes important qualitative study experiences related to recruitment and retention strategies, quantitative assessments of these strategies may also improve our understanding of their correlates and effects. Two prior observational studies have quantitatively examined factors associated with the success of recruitment and retention in intervention studies. Using discriminant function analysis and analysis of variance, Coatsworth et al. [9] found that retention patterns (i.e., non-attenders, variable attenders or consistently high attenders over intervention sessions) were associated with sociodemographic and child- and family-level characteristics in a family-based intervention aiming to 
Table 4 Retention strategies described in articles reviewed

\begin{tabular}{|c|c|c|c|c|c|c|}
\hline \multirow[t]{2}{*}{ Reference } & \multicolumn{4}{|l|}{ Retention strategy } & \multirow[b]{2}{*}{ Participant tracking } & \multirow[t]{2}{*}{ Retention rate } \\
\hline & Intervention design & Incentive & Project bond & Participant convenience & & \\
\hline Davis et al. [15] & Run-in phase & Weekly grocery gift cards & - & Transportation support & - & $79.4 \%(54 / 68)$ \\
\hline \multicolumn{7}{|l|}{ Ventura et al. [16] } \\
\hline Azevedo et al. [17] & - & Rewards for retention & - & - & - & $100 \%(252 / 252)$ \\
\hline Berry et al. [18-20] & $\begin{array}{l}\text { 1) Delayed intervention } \\
\text { for control group } \\
\text { 2) Counseling or } \\
\text { support }\end{array}$ & $\begin{array}{l}\text { 1) Exercise equipment } \\
\text { 2) Money for data } \\
\text { collection } \\
\text { 3) Food } \\
\text { 4) Gifts }\end{array}$ & $\begin{array}{l}\text { 1) Regular contact } \\
\text { 2) Refrigerator magnet } \\
\text { 3) Building staff-participant } \\
\text { relationship }\end{array}$ & $\begin{array}{l}\text { 1) Reminder message } \\
\text { 2) Flexible data collection } \\
\text { days } \\
\text { 3) Childcare } \\
\text { 4) Transportation support }\end{array}$ & $\begin{array}{l}\text { 1) Complete contact } \\
\text { information } \\
\text { 2) Toll-free line } \\
\text { 3) Tracking letter }\end{array}$ & $89.1 \%(638 / 716)$ \\
\hline $\begin{array}{l}\text { Elizondo-Montemayor } \\
\text { et al. [21] }\end{array}$ & - & - & $\begin{array}{l}\text { Building staff-participant } \\
\text { relationship }\end{array}$ & Reminder message & - & $80.8 \%(101 / 125)$ \\
\hline Black et al. [24, 25] & Culturally sensitive & - & - & - & - & $78.3 \%(184 / 235)$ \\
\hline \multicolumn{7}{|l|}{ Hurley et al. [26] } \\
\hline \multicolumn{7}{|l|}{ Witherspoon et al. [27] } \\
\hline Weigensberg et al. [28] & & - & - & $\begin{array}{l}\text { Transportation support } \\
\text { Make-up session }\end{array}$ & - & $82.9 \%(29 / 35)$ \\
\hline Ritchie et al. [33] & \multirow{2}{*}{$\begin{array}{l}\text { 1) Alternative intervention } \\
\text { for control group } \\
\text { 2) Counseling or support } \\
\text { 3) Culturally sensitive }\end{array}$} & \multirow{2}{*}{$\begin{array}{l}\text { 1) Exercise equipment } \\
\text { 2) Recipe books }\end{array}$} & \multirow{2}{*}{$\begin{array}{l}\text { 1) Building staff-participant } \\
\text { relationship } \\
\text { 2) Regular contact }\end{array}$} & \multirow[t]{2}{*}{ Transportation support } & \multirow[t]{2}{*}{-} & \multirow[t]{2}{*}{$57.9 \%(136 / 235$} \\
\hline Sharma et al. [34] & & & & & & \\
\hline Burnet et al. [37] & $\begin{array}{l}\text { 1) Culturally sensitive } \\
\text { 2) Activities at YMCA } \\
\text { and grocery stores }\end{array}$ & - & $\begin{array}{l}\text { Building staff-participant } \\
\text { relationship }\end{array}$ & $\begin{array}{l}\text { 1) Convenient intervention } \\
\text { sites } \\
\text { 2) Transportation support } \\
\text { 3) Child care }\end{array}$ & - & $62.1 \%(18 / 29)$ \\
\hline Davis et al. [38-40] & \multirow[t]{3}{*}{-} & \multirow{3}{*}{$\begin{array}{l}\text { 1) Weekly prizes } \\
\text { 2) Increasing money for } \\
\text { data collections } \\
\text { 3) Food at intervention } \\
\text { session }\end{array}$} & \multirow[t]{3}{*}{ Regular contact } & \multirow[t]{3}{*}{ Transportation support } & \multirow[t]{3}{*}{-} & \multirow[t]{3}{*}{$94.1 \%(209 / 222$} \\
\hline Tkacz et al. [41] & & & & & & \\
\hline Petty et al. [42] & & & & & & \\
\hline Wickham et al. [44] & - & YMCA membership & - & - & - & - \\
\hline Bean et al. [45] & - & $\begin{array}{l}\text { 1) YMCA membership } \\
\text { 2) Grocery store gift } \\
\text { card for data collection }\end{array}$ & - & - & - & - \\
\hline Wysocki et al. $[46,47]$ & $\begin{array}{l}\text { Alternative intervention } \\
\text { for control group }\end{array}$ & $\begin{array}{l}\text { 1) Money for each data } \\
\text { collection } \\
\text { 2) Money for completing } \\
\text { all intervention sessions }\end{array}$ & - & - & - & $96.6 \%(115 / 119)$ \\
\hline Wysocki et al. [48-50] & $\begin{array}{l}\text { Alternative intervention } \\
\text { for control group }\end{array}$ & $\begin{array}{l}\text { 1) Money for each data } \\
\text { collection } \\
\text { 2) Money for completion } \\
\text { of all intervention sessions }\end{array}$ & - & - & - & $88.5 \%(92 / 104)$ \\
\hline
\end{tabular}


Table 4 Retention strategies described in articles reviewed (Continued)

\begin{tabular}{|c|c|c|c|c|c|c|}
\hline Ellis et al. [51, 52] & $\begin{array}{l}\text { Alternative intervention } \\
\text { for control group }\end{array}$ & - & - & $\begin{array}{l}\text { Convenient intervention } \\
\text { sites }\end{array}$ & - & $92.9 \%(118 / 127)$ \\
\hline Story et al. [2] & \multirow{6}{*}{$\begin{array}{l}\text { 1) Alternative intervention } \\
\text { for control group } \\
\text { 2) Fun intervention } \\
\text { activities } \\
\text { 3) Culturally sensitive }\end{array}$} & \multirow{6}{*}{$\begin{array}{l}\text { 1) Gift for intervention } \\
\text { attendance } \\
\text { 2) Money } \\
\text { 3) Increasing money } \\
\text { for data collections } \\
\text { 4) Additional money } \\
\text { for blood draw } \\
\text { 5) Food }\end{array}$} & \multirow{6}{*}{$\begin{array}{l}\text { 1) Family nights } \\
\text { 2) Regular contact } \\
\text { 3) Build relationship } \\
\text { between study and } \\
\text { broader community }\end{array}$} & \multirow{6}{*}{$\begin{array}{l}\text { 1) Convenient intervention } \\
\text { sites } \\
\text { 2) Flexible study procedures } \\
\text { and measurement visits } \\
\text { 3) Home visits for data } \\
\text { collection } \\
\text { 4) Transportation support } \\
\text { 5) Childcare6) Email and } \\
\text { telephone reminders }\end{array}$} & \multirow{6}{*}{$\begin{array}{l}\text { 1) Complete contact } \\
\text { information } \\
\text { 2) Tracking database } \\
\text { 3) Calls from 'non-identifiable' } \\
\text { cell phones }\end{array}$} & \multirow[t]{2}{*}{ Phase l: } \\
\hline Rochon et al. [53] & & & & & & \\
\hline $\begin{array}{l}\text { Kumanyika et al. [54, } \\
55]\end{array}$ & & & & & & $\begin{array}{l}91.4 \%(32 / 35) \text { and } 100 \% \\
(60 / 60)\end{array}$ \\
\hline Klesges et al. $[56,57]$ & & & & & & Phase II: \\
\hline Robinson et al. $[58,59]$ & & & & & & $80.2 \%(243 / 303)$ and \\
\hline Stockton et al. [60] & & & & & & \\
\hline Natale et al. [61] & $\begin{array}{l}\text { Alternative intervention } \\
\text { for control group }\end{array}$ & Incentives (not specified) & Regular contact & - & - & - \\
\hline Nansel et al. [62] & $\begin{array}{l}\text { Alternative intervention } \\
\text { for control group }\end{array}$ & $\begin{array}{l}\text { 1) Money for completing } \\
\text { all data collections } \\
\text { 2) Additional money for } \\
\text { child providing blood } \\
\text { glucose meter data }\end{array}$ & $\begin{array}{l}\text { 1) Appointment } \\
\text { reminder calls } \\
\text { 2) Follow-up calls } \\
\text { after appointment }\end{array}$ & $\begin{array}{l}\text { 1) Transportation support } \\
\text { 2) Midpoint evaluations by } \\
\text { telephone }\end{array}$ & - & $92.3 \%(360 / 390)$ \\
\hline Janicke et al. [63] & \multirow{3}{*}{$\begin{array}{l}\text { 1) Delayed intervention } \\
\text { for control group } \\
\text { 2) Proper participant } \\
\text { goals } \\
\text { 3) Person-centered } \\
\text { intervention }\end{array}$} & \multirow{3}{*}{$\begin{array}{l}\text { 1) Drawing for gift card } \\
\text { at weekly child session } \\
\text { 2) Gift card per family } \\
\text { for each session } \\
\text { 3) Money for data } \\
\text { collections } \\
\text { 4) Food }\end{array}$} & \multirow{3}{*}{$\begin{array}{l}\text { 1) Build community } \\
\text { connections } \\
\text { 2) Regular contact } \\
\text { 3) Phone calls to } \\
\text { participants after } \\
\text { missed sessions }\end{array}$} & \multirow[t]{3}{*}{ Make-up sessions } & \multirow[t]{3}{*}{-} & \multirow[t]{3}{*}{$87.1 \%(81 / 93)$} \\
\hline $\begin{array}{l}\text { Follansbee-Junger } \\
\text { et al. [64] }\end{array}$ & & & & & & \\
\hline Radcliff et al. [65] & & & & & & \\
\hline
\end{tabular}


prevent substance use in adolescent girls. Another study using chi-square analyses found that attrition of adolescent girls (the majority being African Americans) involved in a randomized controlled trial of a HIV-prevention intervention was associated with recruiters' experiences, recruitment method, contact status, and parental awareness of study participation [10]. Our study is the first to examine retention rates quantitatively by participant characteristics and study design in obesity-related trials conducted in minority or low-income children and found results as expected.

In addition to retrospective analysis of the recruitment and retention efforts, prospective studies designed to test specific recruitment and retention strategies are needed. The randomized clinical trial design is considered to provide the strongest causal evidence. We identified three randomized trials that examined the effectiveness of direct mail letters containing different information in the recruitment of minority adults. For example, Brown et al. [11] randomly assigned 30,000 minority women into four groups formed by a factorial design: ethnically specific or generic statement on disease risk and personalized or non-personalized letterhead. They found that women who received letters with the ethnically specific statements were $34 \%$ more likely to respond than women who received letters with a generic statement, while there was no significant difference in response between women who received personalized letters and those who received non-personalized letters. However, we did not identify any randomized controlled trials that examined the effect of recruitment and retention strategies in minority or low-income children. Considering the limited amount of quantitative evidence available, further analytical study is needed to examine the success rates of recruitment and retention strategies in a broader scope.

\section{Limited publications available}

We found that one-third of eligible studies had not published a peer-reviewed paper. This proportion remained true if we allowed for additional time for manuscripts to reach the publication stage by excluding studies that were completed less than 2 years before our search of the CTRD. Ross et al. [12] examined 635 clinical trials funded by the National Institutes of Health and registered within CTRD and found that more than half of the trials did not publish an article in a peer-reviewed journal indexed by Medline within 2.5 years of trial completion. Furthermore, after 51 months of trial completion, a third of trials remained unpublished. Multiple factors might have contributed to this high non-publication rate, including those beyond the control of the investigators $[12,13]$. Ross et al. [12] also suggested that 12-24 months should be the goal for results from clinical trials to be published. Furthermore, among studies with published peer-reviewed papers, the scope and amount of information reported varied. The non-publication of studies and inconsistent report of recruitment and retention hinders the sharing of experiences and lessons learned, as well as limiting the synthesis of data across studies. Reporting guidelines, including STrengthening the Reporting of OBservational studies in Epidemiology (STROBE) and Consolidated Standards of Reporting Trials (CONSORT), have improved the reporting of observational and experimental studies in journals that support these guidelines. The development of guidelines for reporting recruitment and retention would be a first step in improving the quality of information reported in this area.

\section{Strengths and limitations}

An advantage of our study is that we used the CTRD as the study frame and focused specifically on minority or low-income participants. In addition, the studies included varied substantially in terms of participants' characteristics and study design, which allowed us to describe recruitment and retention strategies more broadly and to examine the retention rates quantitatively by study characteristics. Our study has limitations. We searched only one trial registry. However, most obesity-related trials conducted in the United States after the launch of the CTRD might have been registered in this database. In addition, the limited number of studies identified in our study hampered our ability to conduct multivariate analysis, to examine factors associated with retention rates.

\section{Conclusions}

In conclusion, although studies with a published, peerreviewed article generally achieved high retention rates, limited information on recruitment and retention strategies was available. There is a need for more consistent reporting and systematic cataloging of recruitment and retention methods. Both qualitative and quantitative evidence are warranted to inform evidence-based decisions in choosing effective recruitment and retention strategies for trials involving minority or low-income children.

\section{Abbreviations \\ BMI: body mass index; CONSORT: Consolidated Standards of Reporting Trials; CTRD: Clinical Trials Registration Database; GEMS: Girls Health Enrichment Multi-Site Studies; STROBE: STrengthening the Reporting of OBservational studies in Epidemiology.}

\section{Competing interests}

The authors declare that they have no competing interests.

\section{Authors' contributions}

ZC: concept development, literature search, data extraction and analysis, interpretation of results, and manuscript writing and revision. EMS: concept development, data extraction and analysis, interpretation of results, and 
manuscript revision. NES: concept development, interpretation of results, and manuscript revision. MSF and DSW: concept development, interpretation of results, and manuscript writing and revision. All authors have read and approved the final manuscript.

\section{Acknowledgements}

This research was supported by grants U01 HL103561, U01 HL103620, U01 HL103622, U01 HL103629, and U01 HD068890 from the National Heart, Lung, and Blood Institute and the Eunice Kennedy Shriver National Institute of Child Health and Development and the Office of Behavioral and Social Sciences Research. This work was also supported by the National Institute of Diabetes and Digestive and Kidney Diseases at the National Institutes of Health (grant numbers R21DK078239, P30DK050456, P30DK092924). The content is solely the responsibility of the authors and does not necessarily represent the official views of the National Heart, Lung, and Blood Institute, the National Institutes of Health, or the National Institute of Child Health and Development.

\section{Author details}

'Department of Nutrition, Gillings School of Global Public Health, University of North Carolina at Chapel Hill, 2202 McGavran-Greenberg Hall, Campus Box 7461, Chapel Hill, NC 27599, USA. ${ }^{2}$ HealthPartners Institute for Education and Research, Minneapolis, MN, USA.

Received: 25 April 2015 Accepted: 27 November 2015

\section{Published online: 10 December 2015}

\section{References}

1. Probstfield $J$, Frye RL. Strategies for recruitment and retention of participants in clinical trials. JAMA. 2011;306:1798-9.

2. Story M, Sherwood NE, Obarzanek E, Beech BM, Baranowski JC, Thompson NS, et al. Recruitment of African-American pre-adolescent girls into an obesity prevention trial: the GEMS pilot studies. Ethn Dis. 2003;13:S78-87.

3. Schoeppe S, Oliver M, Badland HM, Burke M, Duncan MJ. Recruitment and retention of children in behavioral health risk factor studies: REACH strategies. Int J Behav Med. 2014;21:794-803.

4. Yancey AK, Ortega AN, Kumanyika SK. Effective recruitment and retention of minority research participants. Annu Rev Public Health. 2006;27:1-28.

5. National Institutes of Health. ClinicalTrials.gov Background. http://www clinicaltrials.gov/ct2/about-site/background. Accessed 1 Dec 2014.

6. International Committee of Medical Journal Editors. Clinical Trials Registration. http://www.icmje.org/about-icmje/faqs/clinical-trialsregistration/. Accessed 1 Dec 2014.

7. International Committee of Medical Journal Editors. Journals Following the ICMJE Recommendations. http://www.icmje.org/journals-following-theicmje-recommendations/. Accessed 1 Dec 2014.

8. Amon KL, Campbell AJ, Hawke C, Steinbeck K. Facebook as a recruitment tool for adolescent health research: a systematic review. Acad Pediatr. 2014; 14:439-47.

9. Coatsworth JD, Duncan LG, Pantin H, Szapocznik J. Retaining ethnic minority parents in a preventive intervention: the quality of group process. J Prim Prev. 2006;27:367-89.

10. Seibold-Simpson S, Morrison-Beedy D. Avoiding early study attrition in adolescent girls: impact of recruitment contextual factors. West J Nurs Res. 2010;32:761-78.

11. Brown SD, Lee K, Schoffman DE, King AC, Crawley LM, Kiernan M. Minority recruitment into clinical trials: experimental findings and practical implications. Contemp Clin Trials. 2012;33:620-3.

12. Ross JS, Tse T, Zarin DA, Xu H, Zhou L, Krumholz HM. Publication of NIH funded trials registered in ClinicalTrials.gov: cross sectional analysis. BMJ. 2012;344:d7292.

13. Hudson KL, Collins FS. Sharing and reporting the results of clinical trials. JAMA. 2015;313:355-6.

14. Hasson RE, Adam TC, Davis JN, Kelly LA, Ventura EE, Byrd-Williams CE, et al. Randomized controlled trial to improve adiposity, inflammation, and insulin resistance in obese African-American and Latino youth. Obesity (Silver Spring). 2012;20:811-8.

15. Davis JN, Kelly LA, Lane CJ, Ventura EE, Byrd-Williams CE, Alexandar KA, et al. Randomized control trial to improve adiposity and insulin resistance in overweight Latino adolescents. Obesity (Silver Spring). 2009;17:1542-8.

16. Ventura E, Davis J, Byrd-Williams C, Alexander K, McClain A, Lane CJ, et al. Reduction in risk factors for type 2 diabetes mellitus in response to a low- sugar, high-fiber dietary intervention in overweight Latino adolescents. Arch Pediatr Adolesc Med. 2009;163:320-7.

17. Azevedo KJ, Mendoza S, Fernandez M, Haydel KF, Fujimoto M, Tirumalai EC, et al. Turn off the TV and dance! Participation in culturally tailored health interventions: implications for obesity prevention among Mexican American girls. Ethn Dis. 2013;23:452-61.

18. Berry DC, Neal M, Hall EG, McMurray RG, Schwartz TA, Skelly AH, et al. Recruitment and retention strategies for a community-based weight management study for multi-ethnic elementary school children and their parents. Public Health Nurs. 2013;30:80-6.

19. Berry DC, Schwartz TA, McMurray RG, Skelly AH, Neal M, Hall EG, et al. The family partners for health study: a cluster randomized controlled trial for child and parent weight management. Nutr Diabetes. 2014;4:e101.

20. Berry DC, McMurray R, Schwartz TA, Skelly A, Sanchez M, Neal M, et al. Rationale, design, methodology and sample characteristics for the family partners for health study: a cluster randomized controlled study. BMC Public Health. 2012;12:250. 2458-12-250.

21. Elizondo-Montemayor L, Moreno-Sanchez D, Gutierrez NG, MonsivaisRodriguez F, Martinez U, Lamadrid-Zertuche AC, et al. Individualized tailormade dietetic intervention program at schools enhances eating behaviors and dietary habits in obese Hispanic children of low socioeconomic status. Sci World J. 2014;2014:484905.

22. Wang Y, Tussing L, Odoms-Young A, Braunschweig C, Flay B, Hedeker D, et al. Obesity prevention in low socioeconomic status urban African-American adolescents: study design and preliminary findings of the HEALTH-KIDS study. Eur J Clin Nutr. 2006;60:92-103.

23. Wang Y, Li J, Caballero B. Resemblance in dietary intakes between urban low-income African-American adolescents and their mothers: the healthy eating and active lifestyles from school to home for kids study. J Am Diet Assoc. 2009;109:52-63.

24. Black MM, Hager ER, Le K, Anliker J, Arteaga SS, Diclemente C, et al. Challenge! Health promotion/obesity prevention mentorship model among urban, black adolescents. Pediatrics. 2010;126:280-8.

25. Black MM, Arteaga SS, Sanders J, Hager ER, Anliker JA, Gittelsohn J, et al. College mentors: a view from the inside of an intervention to promote health behaviors and prevent obesity among low-income, urban, African American adolescents. Health Promot Pract. 2012;13:238-44.

26. Hurley KM, Oberlander SE, Merry BC, Wrobleski MM, Klassen AC, Black MM The healthy eating index and youth healthy eating index are unique, nonredundant measures of diet quality among low-income, African American adolescents. J Nutr. 2009;139:359-64.

27. Witherspoon D, Latta L, Wang Y, Black MM. Do depression, self-esteem, body-esteem, and eating attitudes vary by BMI among African American adolescents? J Pediatr Psychol. 2013;38:1112-20.

28. Weigensberg MJ, Lane CJ, Avila Q, Konersman K, Ventura E, Adam T. Imagine HEALTH: results from a randomized pilot lifestyle intervention for obese Latino adolescents using Interactive Guided Imagery ${ }^{\text {SM }}$. BMC Complement Altern Med. 2014;14:28. 6882-14-28.

29. Wilson DK, Van Horn ML, Kitzman-Ulrich H, Saunders R, Pate R, Lawman HG, et al. Results of the 'active by choice today' (ACT) randomized trial for increasing physical activity in low-income and minority adolescents. Health Psychol. 2011;30:463-71.

30. Wilson DK, Kitzman-Ulrich H, Williams JE, Saunders R, Griffin S, Pate R, et al. An overview of 'the active by choice today' (ACT) trial for increasing physical activity. Contemp Clin Trials. 2008;29:21-31.

31. Wilson DK, Lawman HG, Segal M, Chappell S. Neighborhood and parental supports for physical activity in minority adolescents. Am J Prev Med. 2011; 41:399-406.

32. Naar-King S, Ellis D, Kolmodin K, Cunningham P, Jen KL, Saelens B, et al. A randomized pilot study of multisystemic therapy targeting obesity in African-American adolescents. J Adolesc Health. 2009;45:417-9.

33. Ritchie LD, Sharma S, Ikeda JP, Mitchell RA, Raman A, Green BS, et al. Taking action together: a YMCA-based protocol to prevent type-2 diabetes in highBMI inner-city African American children. Trials. 2010;11:60. 6215-11-60.

34. Sharma S, Fleming SE. One-year change in energy and macronutrient intakes of overweight and obese inner-city African American children: effect of community-based taking action together type 2 diabetes prevention program. Eat Behav. 2012;13:271-4.

35. Eisenmann JC, Alaimo K, Pfeiffer K, Paek HJ, Carlson JJ, Hayes H, et al. Project FIT: rationale, design and baseline characteristics of a school- and community-based intervention to address physical activity and healthy 
eating among low-income elementary school children. BMC Public Health. 2011;11:607. 2458-11-607

36. Barkin SL, Gesell SB, Po'e EK, Escarfuller J, Tempesti T. Culturally tailored, family-centered, behavioral obesity intervention for Latino-American preschool-aged children. Pediatrics. 2012;130:445-56.

37. Burnet DL, Plaut AJ, Wolf SA, Huo D, Solomon MC, Dekayie G, et al. Reachout: a family-based diabetes prevention program for African American youth. J Natl Med Assoc. 2011;103:269-77.

38. Davis CL, Tomporowski PD, Boyle CA, Waller JL, Miller PH, Naglieri JA, et al. Effects of aerobic exercise on overweight children's cognitive functioning: a randomized controlled trial. Res Q Exerc Sport. 2007;78:510-9.

39. Davis CL, Tomporowski PD, McDowell JE, Austin BP, Miller PH, Yanasak NE, et al. Exercise improves executive function and achievement and alters brain activation in overweight children: a randomized, controlled trial. Health Psychol. 2011;30:91-8.

40. Davis CL, Pollock NK, Waller JL, Allison JD, Dennis BA, Bassali R, et al. Exercise dose and diabetes risk in overweight and obese children: a randomized controlled trial. JAMA. 2012;308:1103-12.

41. Tkacz J, Young-Hyman D, Boyle CA, Davis CL. Aerobic exercise program reduces anger expression among overweight children. Pediatr Exerc Sci. 2008;20:390-401.

42. Petty KH, Davis CL, Tkacz J, Young-Hyman D, Waller JL. Exercise effects on depressive symptoms and self-worth in overweight children: a randomized controlled trial. J Pediatr Psychol. 2009;34:929-39.

43. Madsen K, Thompson H, Adkins A, Crawford Y. School-community partnerships: a cluster-randomized trial of an after-school soccer program. JAMA Pediatr. 2013;167:321-6.

44. Wickham EP, Stern M, Evans RK, Bryan DL, Moskowitz WB, Clore JN, et al. Prevalence of the metabolic syndrome among obese adolescents enrolled in a multidisciplinary weight management program: clinical correlates and response to treatment. Metab Syndr Relat Disord. 2009;7:179-86.

45. Bean MK, Mazzeo SE, Stern M, Evans RK, Bryan D, Ning Y, et al. Six-month dietary changes in ethnically diverse, obese adolescents participating in a multidisciplinary weight management program. Clin Pediatr (Phila). 2011;50: 408-16.

46. Wysocki T, Harris MA, Greco P, Bubb J, Danda CE, Harvey LM, et al. Randomized, controlled trial of behavior therapy for families of adolescents with insulin-dependent diabetes mellitus. J Pediatr Psychol. 2000;25:23-33.

47. Wysocki T, Greco P, Harris MA, Bubb J, White NH. Behavior therapy for families of adolescents with diabetes: maintenance of treatment effects. Diabetes Care. 2001;24:441-6.

48. Wysocki T, Harris MA, Buckloh LM, Mertlich D, Lochrie AS, Taylor A, et al. Effects of behavioral family systems therapy for diabetes on adolescents' family relationships, treatment adherence, and metabolic control. J Pediatr Psychol. 2006;31:928-38.

49. Wysocki T, Harris MA, Buckloh LM, Mertlich D, Lochrie AS, Mauras N, et al. Randomized trial of behavioral family systems therapy for diabetes: maintenance of effects on diabetes outcomes in adolescents. Diabetes Care. 2007;30:555-60.

50. Wysocki T, Harris MA, Buckloh LM, Mertlich D, Lochrie AS, Taylor A, et al. Randomized, controlled trial of behavioral family systems therapy for diabetes: maintenance and generalization of effects on parent-adolescent communication. Behav Ther. 2008;39:33-46.

51. Ellis DA, Frey MA, Naar-King S, Templin T, Cunningham P, Cakan N. Use of multisystemic therapy to improve regimen adherence among adolescents with type 1 diabetes in chronic poor metabolic control: a randomized controlled trial. Diabetes Care. 2005:28:1604-10.

52. Ellis D, Naar-King S, Templin T, Frey M, Cunningham P, Sheidow A, et al. Multisystemic therapy for adolescents with poorly controlled type 1 diabetes: reduced diabetic ketoacidosis admissions and related costs over 24 months. Diabetes Care. 2008;31:1746-7.

53. Rochon J, Klesges RC, Story M, Robinson TN, Baranowski T, Obarzanek E, et al. Common design elements of the Girls Health Enrichment Multi-site Studies (GEMS). EthnDis. 2003;13:S6; 14.

54. Kumanyika SK, Story M, Beech BM, Sherwood NE, Baranowski JC, Powell TM, et al. Collaborative planning for formative assessment and cultural appropriateness in the girls health enrichment multi-site studies (GEMS): a retrospection. Ethn Dis. 2003;13:S15-29.

55. Kumanyika S, Fassbender J, Phipps E, Tan-Torres S, Localio R, Morales KH, et al. Design, recruitment and start up of a primary care weight loss trial targeting African American and Hispanic adults. Contemp Clin Trials. 2011; 32:215-24.

56. Klesges RC, Obarzanek E, Klesges LM, Stockton MB, Beech BM, Murray DM, et al. Memphis girls health enrichment multi-site studies (GEMS). Phase 2: design and baseline. Contemp Clin Trials. 2008;29:42-55.

57. Klesges RC, Obarzanek E, Kumanyika S, Murray DM, Klesges LM, Relyea GE, et al. The Memphis girls' health enrichment multi-site studies (GEMS): an evaluation of the efficacy of a 2-year obesity prevention program in African American girls. Arch Pediatr Adolesc Med. 2010;164:1007-14.

58. Robinson TN, Matheson DM, Kraemer HC, Wilson DM, Obarzanek E, Thompson NS, et al. A randomized controlled trial of culturally tailored dance and reducing screen time to prevent weight gain in low-income African American girls: Stanford GEMS. Arch Pediatr Adolesc Med. 2010;164 995-1004.

59. Robinson TN, Kraemer HC, Matheson DM, Obarzanek E, Wilson DM, Haskell WL, et al. Stanford GEMS phase 2 obesity prevention trial for low-income African-American girls: design and sample baseline characteristics. Contemp Clin Trials. 2008;29:56-69.

60. Stockton MB, McClanahan BS, Lanctot JQ, Klesges RC, Beech BM. Identification of facilitators and barriers to participation in weight gain prevention research by African American girls. Contemp Clin Trials. 2012;33:38-45.

61. Natale R, Scott SH, Messiah SE, Schrack MM, Uhlhorn SB, Delamater A. Design and methods for evaluating an early childhood obesity prevention program in the childcare center setting. BMC Public Health. 2013;13:78. 2458-13-78.

62. Nansel TR, lannotti RJ, Liu A. Clinic-integrated behavioral intervention for families of youth with type 1 diabetes: randomized clinical trial. Pediatrics. 2012;129:e866-73.

63. Janicke DM, Sallinen BJ, Perri MG, Lutes LD, Huerta M, Silverstein JH, et al Comparison of parent-only vs family-based interventions for overweight children in underserved rural settings: outcomes from project STORY. Arch Pediatr Adolesc Med. 2008;162:1119-25.

64. Follansbee-Junger $\mathrm{K}$, Janicke DM, Sallinen BJ. The influence of a behavioral weight management program on disordered eating attitudes and behaviors in children with overweight. J Am Diet Assoc. 2010;110:1653-9.

65. Radcliff TA, Bobroff LB, Lutes LD, Durning PE, Daniels MJ, Limacher MC, et al. Comparing costs of telephone vs face-to-face extended-care programs for the management of obesity in rural settings. J Acad Nutr Diet. 2012;112: $1363-73$.

\section{Submit your next manuscript to BioMed Central and we will help you at every step:}

- We accept pre-submission inquiries

- Our selector tool helps you to find the most relevant journal

- We provide round the clock customer support

- Convenient online submission

- Thorough peer review

- Inclusion in PubMed and all major indexing services

- Maximum visibility for your research

Submit your manuscript at www.biomedcentral.com/submit
C) BioMed Central 